\title{
COMPARISON AND SUITABILITY ASSESSMENT OF SLOPE CALCULATION ALGORITHMS ON DIFFERENT TERRAINS USING AERIAL SURVEY
}

\author{
Y. Singh*, S. Bhandari, D. Tamang, A. Basnet, R. Shrestha, B. Awasthi \\ Department of Geomatics Engineering, Kathmandu University, Nepal - yash420g@gmail.com, bhandarisurace18@gmail.com, \\ mtnekros@gmail.com, aashishbasnet46@gmail.com, reshma@ku.edu.np, basantawasthi8@gmail.com
}

Commission V, WG V/7 \& Commission IV, WG IV/6

KEY WORDS: ANOVA, Post-Hoc, IBM SPSS, Slope Calculation Algorithms, Aerial Survey, QGIS

\begin{abstract}
:
Digital Elevation Models are one of the important datasets of any Geographic Information System (GIS) and so are the parameters derived from them. One such parameter is slope, whose accuracy can have a significant effect on many engineering and construction works. This paper addresses the eight-slope calculation methods that are currently available to calculate slope value from a DEM and compares how these methods works on different slope range and values. These methods were applied to calculate slope from DEM of $30 \mathrm{~m}$. To determine the method that calculates the most accurate slope value for a particular slope range by comparing them with actual slope value is the main objective of this paper. The methods 2FD, 3FD, 3FDWRD, Average Neighborhood, Constrained Quadratic Surface and FFD has given similar results across all slope range while the algorithms that appears to yield the most varying results are Maximum Max and Simple D. In addition, it is observed that the choice of algorithms is more important when grade slope is less than 10 percent. However, for terrains with above 10 percent slope, the choice of algorithms seems less important with only a difference of approximately 0.5 gradient.
\end{abstract}

\section{INTRODUCTION}

Geographic Information Systems (GIS) offer a cost-effective way to analyze and inventory Digital Elevation Model (DEM). Aerial survey is one of the many ways to obtain field data for the DEM generation and has been used during the research. Different parameters can be derived from DEM, and one such parameter that has been used in this project is slope. Slope is a metric that is essential to describe surface processes, including overland flow, sediment transport and soil erosion and needed during locating a hydropower plant. The application of slope is endless. The accuracy with which the slope of an area is calculated and used for any projects can have an impact on the output of those project.

There are several different mathematical computational algorithms used to calculate slope from a DEM. The analysis of the different slope calculation methods is an essential as it may create vast difference in the final output of any sort of project whose foundation is laid on the slope value. Every slope calculation method is different from the other and its working mechanism may suit for certain slope range over others. Eight Slope calculation methods has been used in the project to calculate the slope from DEM. All eight algorithms are developed using different techniques and considerations so the question arises whether they give different slope values while calculating slopes of different terrains or they give same results across different terrain surfaces? Therefore, this project compares the results of the slopes generated applying different algorithms using statistical testing and assess the suitability of each algorithms in different classified slope surfaces. Among many methods of slope calculation, the ArcGIS uses the Average neighborhood method to calculate the slope from DEM. It uses a $3 * 3$ cell size and the cell window contains eight neighboring elevations.

\footnotetext{
* Corresponding author
}

\subsection{Slope Calculation Methods}

At every point in a DEM the slope can be defined as a function of gradients in the $\mathrm{X}$ and $\mathrm{Y}$ direction:

Sloperadian $=\arctan \sqrt{\mathrm{f}_{\mathrm{x}}{ }^{2}+\mathrm{f}_{\mathrm{y}}{ }^{2}}$

Where, $\mathrm{fx}$ is slope in $\mathrm{x}$-direction and

$$
\text { fy }=\text { slope in } y \text {-direction }
$$

The key in slope estimation is the computation of the perpendicular gradients fx and fy. Different algorithms, using different techniques to calculate $\mathrm{fx}$ and fy yield the diversity in estimated slope. The common approach when estimating $\mathrm{fx}$ and fy is by using a moving $3 \times 3$ window to derive the finite differential or local polynomial surface fit for the calculation. Methods 1, 3 and 4 are methods based on approximation of differential operators by finite differences. Method 2 compares the central elevations with its eight neighbors, adopting the largest. Eight methods used for calculating slope from DEM obtained after processing of images obtained from aerial survey are:

All these methods work on a moving window of DEM. Let's assume the cell size of the DEM be $\mathrm{g}$.

\begin{tabular}{|l|l|l|}
\hline 9 & 8 & 7 \\
\hline 6 & 5 & 4 \\
\hline 3 & 2 & 1 \\
\hline
\end{tabular}

Figure $1.3 \times 3$ window with numbered cells 
In the following mathematical equations of slope, $\mathrm{zi}(\mathrm{i}=1,2, .$. 9) is the elevation value in cell $\mathrm{i}$.

1.1.1 Second-order finite difference (2FD): The rate of change in the $\mathrm{x}$ and $\mathrm{y}$-direction for the central cell or cell 5 is given by:

$\mathrm{fx}=(\mathrm{z} 6-\mathrm{z} 4) / 2 \mathrm{~g} ; \quad \mathrm{fy}=(\mathrm{z} 8-\mathrm{z} 2) / 2 \mathrm{~g}$

Where $z 2, z 4, z 6$ and $z 8$ are the elevation values of cell $2,4,6$,

$$
\mathrm{g}=\text { cell size. }
$$

1.1.2 Maximum Max (Downhill slope) (Dr. Ashraf, 2012). The slope of the central cell or cell 5 is calculated by:

Slope $=\arctan \left[\max \left(\frac{\mathrm{z} 5-\mathrm{zj}}{\mathrm{Lj}}\right)\right]$

Where $\mathrm{j}=1,2,3,4,6,7,8,9$

$L_{j}=\Delta x=g$, when $\mathrm{j}=2,4,6,8$ in the orthogonal direction; $L j=\Delta x \times \sqrt{2}=g \times \sqrt{2}$, when $\mathrm{j}=1,3,7$ and 9 in the diagonal direction.

1.1.3 Simple diff erence (Simple-D) (Jones, 1998). The rate of change in the $\mathrm{x}$ and $\mathrm{y}$ direction for the central cell or cell 5 is given by:

$\mathrm{fx}=(\mathrm{z} 5-\mathrm{z} 4) / \mathrm{g} ; \mathrm{fy}=(\mathrm{z} 5-\mathrm{z} 2) / \mathrm{g}$

Where, z5, z4 and z2 are elevations of cell 5,4 and 2 respectively and $\mathrm{g}=$ cell size

1.1.4 Average Neighborhood (ArcGIS Algorithm) (Horn, 1981). The rate of change in the $\mathrm{x}$ and $\mathrm{y}$ direction for the central cell or cell 5 is calculated with the following formula: $\mathrm{fx}=(\mathrm{z} 3-\mathrm{z} 1+2(\mathrm{z} 6-\mathrm{z} 4)+\mathrm{z} 9-\mathrm{z} 7) / 8 \mathrm{~g}$

$f y=(z 7-z 1+2(z 8-z 2)+z 9-z 3) / 8 g$

Where, $\mathrm{z} 1, \mathrm{z} 2, \mathrm{z} 3$ and $\mathrm{z} 7, \mathrm{z} 8, \mathrm{z} 9$ are elevations of cell 1, 2, 3 and $7,8,9$ respectively and $g=$ cell size

1.1.5 Three-order Finite Difference Weighted by Reciprocal of Distance (3FDWRD) (Unwin, 1981). The formulas for the slope calculation using this method is as below:

$\mathrm{fx}_{\mathrm{x}}=(\mathrm{z} 3-\mathrm{z} 1+\sqrt{2}(\mathrm{z} 6-\mathrm{z} 4)+\mathrm{z} 9-\mathrm{z} 7) /(4+2 \sqrt{2}) \mathrm{g}$

$f y=(z 7-z 1+\sqrt{2}(z 8-z 2)+z 9-z 3) /(4+2 \sqrt{2}) g$

1.1.6 Three-order Finite Difference, Linear regression plan (3FD) (Sharpnack \& A. and AKin, 1969). The formulas for the slope calculation using this method is as below:

$f x=(z 3-z 1+z 6-z 4+z 9-z 7) / 6 g ; f y=(z 7-z 1+z 8-z 2+z 9-z 3) / 6 g$

1.1.7 Frame Finite difference (FFD) (Chu \& Tsai, 1995). The formulas for the slope calculation using this method is as below:

$f x=(z 3-z 1+z 9-z 7) / 4 g ; f y=(z 7-z 1+z 9-z 3) / 4 g$

1.1.8 Constrained Quadratic Surface Quad Surface (Wood, 1996)

$\mathrm{F}(\mathrm{x}, \mathrm{y})=\mathrm{ax}^{2}+\mathrm{by}^{2}+\mathrm{cxy}+\mathrm{dx}+\mathrm{ey}+\mathrm{f} ; \mathrm{AX}=\mathrm{Z}=\mathrm{F}(\mathrm{x}, \mathrm{y})$

Where, A has been defined (see fig. (4)), X stands for unknown vector of parameters (see fig.(3)) and $Z$ is the elevation vector (see fig. (2)). The number of equations is more than the unknown parameters, so there is no "true" solution. We use the least-squares method to determine the indices of the constrained quadratic surface.

$\mathrm{A}^{\mathrm{T}} \mathrm{AX}=\mathrm{A}^{\mathrm{T}} \mathrm{Z} ; \mathrm{X}=\left(\mathrm{A}^{\mathrm{T}} \mathrm{A}\right)^{-1} \mathrm{~A}^{\mathrm{T}} \mathrm{Z}$

It is then relatively easy to estimate the $\mathrm{fx}$ and fy values at the center of the $3 \times 3$ window.

$\mathrm{fx} \mid \mathrm{x}=0, \mathrm{y}=0=\mathrm{d}$

fy $\mid x=0, y=0=e$

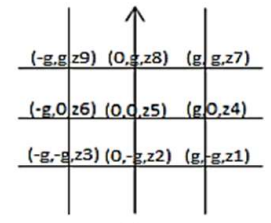

(1)

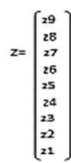

(2)

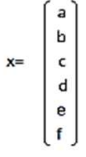

(3)

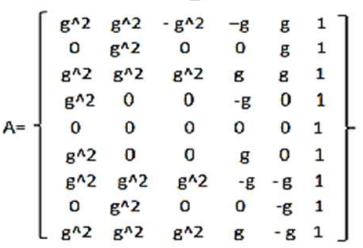

(4)
Figure 2. Parameters for slope calculation

\subsection{ANOVA Test}

An ANOVA test is a way to find out if survey or experiment results are significant. In other words, it helps to determine whether to reject the null hypothesis or accept the alternate hypothesis. Basically, while testing groups to see if there's a difference between them (Stephanie, 2019).

1.2.1 One-Way ANOVA: The One-Way ANOVA ("analysis of variance") compares the means of two or more independent groups in order to determine whether there is statistical evidence that the associated population means are significantly different. One-Way ANOVA is a parametric test. The variables used in this test are known as: Dependent variable. (slope) and Factor (algorithm)

1.2.2 Level of Significance: The level of significance is defined as the probability of rejecting a null hypothesis by a test when it is really true. Generally, it is denoted by $\alpha$ and should be as low as possible. For our project, level of significance that we used is 0.05 .

1.2.3 Confidence Level: Confidence level is an index of surety in the data. It is expressed in percentage and it is defined $95 \%$ for our project. $95 \%$ confidence level indicates can be $95 \%$ certain that it contains the true mean of the population. This $95 \%$ confidence will ascertain that we are accounting for $95 \%$ of the possible results and uncertain about $5 \%$ which may be due to random errors.

\subsection{Post Hoc Test}

Post hoc tests are designed for situations in which the researcher has already obtained a significant omnibus F-test with a factor that consists of three or more means and additional exploration of the differences among means is needed to provide specific information on which means are significantly different from each other (Stevens, 1999). 


\section{METHODOLOGY}

\subsection{Study Area}

The study area for the project was chosen to be within the premises of Kathmandu University, Dhulikhel, Nepal. The study area consists of varying slopes and land cover. The area of the study area is $97335.51 \mathrm{~m}^{2}$. The study area was selected so as to meet the project objectives.

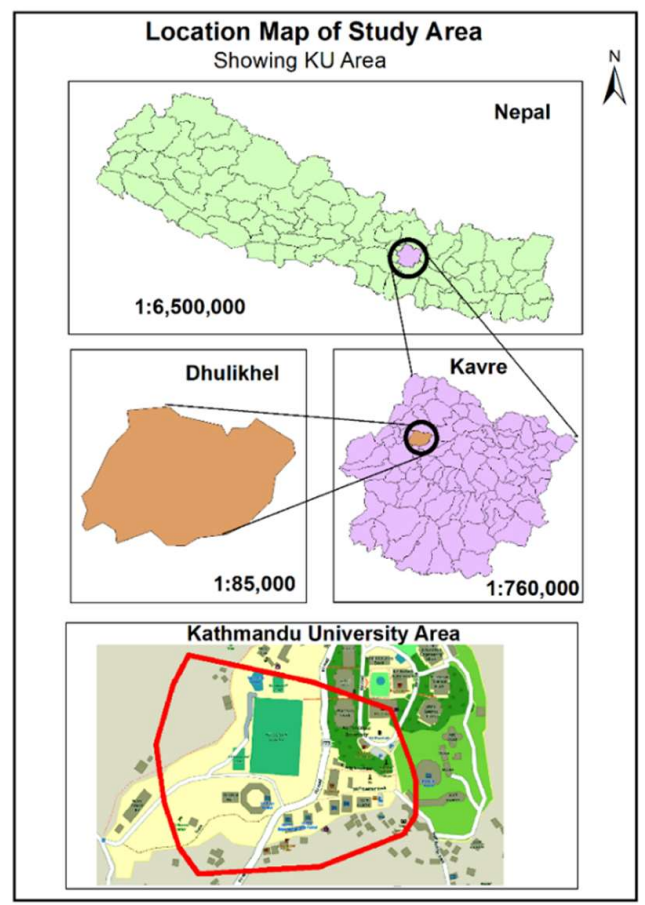

Figure 3. Study Area Map

\subsection{Workflow}

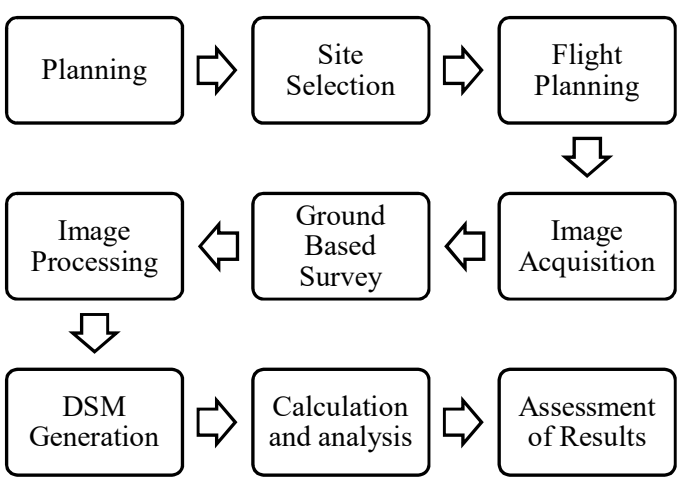

Figure 4. Workflow diagram

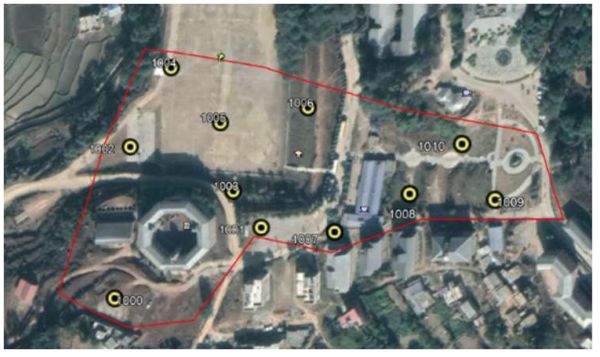

Figure 5. GCP selection for study area

2.2.1 Image Processing: Different software are available for processing of images captured from drone survey. DTM, DEM and DSM can be generated using such software. The reports of the processing can be obtained after the processing. Pix4D mapper was used for the image processing.

2.2.2 DTM Generation: Digital Terrain Model is generated after processing the images. DTM is further used for analysis and derivation of results.

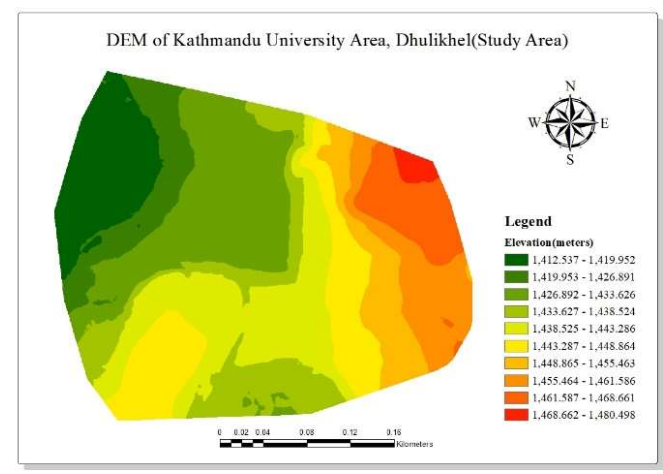

Figure 6. DTM of study area

2.2.3 Calculation and Analysis: Depending upon the range of slopes, the algorithms can produce different results. Thus, the terrain was classified into six different groups based on slope range. The purpose was to make the result of the analysis applicable for general purposes. Owing to this, the slopes were divided into continuous interval of slopes.

Table 1. Slope Classification

\begin{tabular}{|l|l|l|}
\hline Class & Type & Description \\
\hline 1 & Little or none & Little or no slope: 0 - 4 \% gradient. \\
\hline 2 & Gentle & Gentle slopes: 4 - 10 \% gradient. \\
\hline 3 & Moderate & $\begin{array}{l}\text { Moderate slopes: 10 - 15 \% } \\
\text { gradient. }\end{array}$ \\
\hline 4 & Steep & Steep slopes: 15 - 30 \% gradient. \\
\hline 5 & $\begin{array}{l}\text { Extremely } \\
\text { steep }\end{array}$ & $\begin{array}{l}\text { Extremely steep slopes: 30 - 60\% } \\
\text { gradient. }\end{array}$ \\
\hline 6 & $\begin{array}{l}\text { Excessively } \\
\text { steep }\end{array}$ & $\begin{array}{l}\text { Excessively steep slopes: }>60 \% \\
\text { gradient. }\end{array}$ \\
\hline
\end{tabular}

All eight algorithms are developed using different techniques and considerations so the question arises whether they give 
different values while calculating slopes of different terrains or they give same results across different terrain surfaces.

To decide this, we considered a hypothesis test with null and alternate hypothesis as:

Null: The final slopes are not affected by the algorithms used Alternate: The final slopes depend on the algorithms used.

Analysis of variance (ANOVA) is a statistical technique that is used to check if the means of two or more groups are significantly different from each other. ANOVA checks the impact of one or more factors by comparing the means of different samples (Singh, 2018). ANOVA test can be computed manually but for small volume of the data only. But the data we work ranges up to 250000 for a single slope class. Thus, used IBM SPSS Statistics to conduct ANOVA test.

$95 \%$ confidence interval and level of significance as 0.05 was considered. It indicates if the level of significance after applying ANOVA test is less than 0.05 then the null hypothesis is rejected and accept alternate hypothesis which concludes that slopes are dependent on the algorithms used. Major components involved in ANOVA test are within group variability and between group variability.

After deciding whether the means of the slopes from different algorithms are significantly different or not, it is followed by Post Hoc test. The Post Hoc test provides the difference between the mean, standard error and significance between the mean slopes calculated from different algorithms. Similarly, after the Post Hoc the Means Plot provides visualizations to the Post Hoc test

2.2.4 Accuracy Analysis of slopes with ground calculated slopes: Ground survey was conducted using Total Station to calculate the Ground slopes that was further used to validate the slopes calculated after applying the algorithms on classified slope divisions. The sample points required to validate the slope were calculated considering the rules for the sample calculation. The sample areas on the ground were defined for all six slope classes in the extent that they meet the sampling rule. The elevation data were taken within the sample area and slopes were calculated from the elevation data and used as the reference value for the suitability assessment of the algorithms for each slope class. Algorithm with the lowest RMSE error will be the most accurate method for that specific slope class. Each sample area on the ground includes area of $\left(15^{*} 15\right) \mathrm{m}^{2}$.

To check the accuracy of the slopes calculated using all eight algorithms, a ground survey was carried out using Total Station. For all six slope classes, sample points were calculated using the sample calculator. The sample area on the ground was selected so as to meet the sampling rule and required sample points. The sample areas each covered an area of $15 \mathrm{~m} * 15 \mathrm{~m}$ on the ground. The sample areas were staked out on the ground and elevation data within the sample area were observed and further processed through GIS and slopes for each class were calculated. The mean slope for each sample area was considered the most probable value of the slope for that slope class. The mean of the sample was used to tally with the mean slope calculated using algorithms. The RMSE was used as the measure to analyze the slope accuracy.

\section{RESULT}

ANOVA Test for Class 1: The ANOVA test was significance which indicates that at least two algorithms among the eight algorithms were different for Class 1.

Table 2. ANOVA Test Result of Class 1

\begin{tabular}{|l|r|r|r|r|r|}
\hline & \multicolumn{1}{|c|}{$\begin{array}{c}\text { Sum of } \\
\text { Squares }\end{array}$} & \multicolumn{1}{c|}{ df } & Mean Square & F & Sig. \\
\hline Between Groups & 88411.336 & 7 & 12630.191 & 5590.267 & .000 \\
Within Groups & 395886.752 & 175224 & 2.259 & & \\
Total & 484298.089 & 175231 & & & \\
\hline
\end{tabular}

Post Hoc Test Class 1: Following the ANOVA test, Post-Hoc test was done to find out the difference among the different algorithms. The following table from Tukey's Post-Hoc test show that the slopes calculated using Maximum Max and Simple D was significantly different from all other algorithms with the level of significance of 1.00. 3FD, 3FDWRD, Average Neighborhood, FFD didn't produce significantly different results from each other. FFD, Constrained Quadratic Surface and 2FD also didn't had significantly different result from one another.

Table 3. Tukey Test Result of Class 1

\begin{tabular}{|l|c|c|c|c|c|}
\hline & & \multicolumn{5}{|c|}{ Subset for alpha $=0.05$} \\
\cline { 3 - 6 } Algorithm & $\mathrm{N}$ & 1.000 & 2.000 & 3.000 & 4.000 \\
\hline 3FD & 21904 & 1.381 & & & \\
3FDWRD & 21904 & 1.383 & & & \\
AvgNeighbourhood & 21904 & 1.385 & & & \\
FFD & 21904 & 1.394 & 1.394 & & \\
ConstrainedQuadSurface & 21904 & 1.416 & 1.416 & & \\
2FD & 21904 & & 1.434 & & \\
SimpleD & 21904 & & & 2.983 & \\
MaximumMax & 21904 & & & & 3.092 \\
Sig. & & .242 & .107 & 1.000 & 1.000 \\
\hline
\end{tabular}

\section{Mean plot of Class 1:}

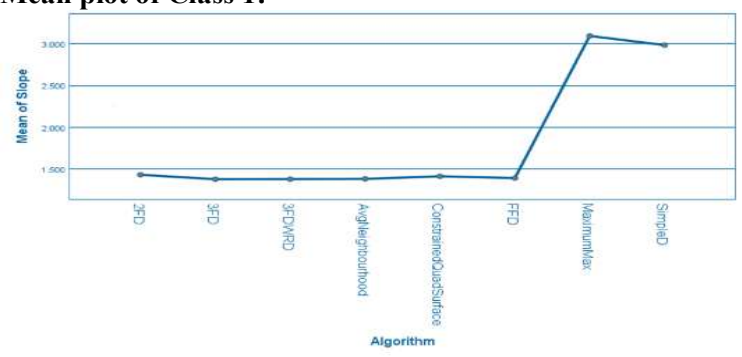

Figure 7. Mean slopes of each algorithm for class 1

ANOVA Test for Class 2: The ANOVA test was significance which indicates that at least two algorithms among the eight algorithms were different for Class 2 .

Table 4. ANOVA Test Result of Class 2

\begin{tabular}{|l|c|r|r|r|r|}
\hline & \multicolumn{1}{|c|}{$\begin{array}{c}\text { Sum of } \\
\text { Squares }\end{array}$} & \multicolumn{1}{c|}{ df } & Mean Square & F & Sig. \\
\hline Between Groups & 68051.924 & 7 & 9721.703 & 134.864 & .000 \\
Within Groups & 12631037.961 & 175224 & 72.085 & & \\
Total & 12699089.885 & 175231 & & & \\
\hline
\end{tabular}

Post Hoc Test Class 2: Following ANOVA test, post-hoc test was done to find out the difference among the different algorithms. The following table from Tukey's Post-Hoc test 
show that the slopes calculated using Maximum Max and Simple D was significantly different from all other algorithms with mean values, significantly higher than the rest of the algorithms while 3FD, FFD, 3FDWRD, Average Neighborhood, Constrained Quadratic Surface and 2FD didn't produce significantly different results.

Table 5. Tukey Test Result of Class 2

\begin{tabular}{|l|c|c|c|c}
\hline & & \multicolumn{3}{|c}{ Subset for alpha $=0.05$} \\
\cline { 3 - 5 } Algorithm & $\mathrm{N}$ & 1 & 2 & 3 \\
\hline FFD & 21904 & 9.377 & & \\
3FD & 21904 & 9.379 & & \\
3FDWRD & 21904 & 9.381 & & \\
AvgNeighbourhood & 21904 & 9.385 & & \\
ConstrainedQuadSurface & 21904 & 9.396 & & \\
2FD & 21904 & 9.418 & & \\
SimpleD & 21904 & & 10.096 & \\
MaximumMax & 21904 & & & 11.240 \\
Sig. & & 1.000 & 1.000 & 1.000 \\
\hline
\end{tabular}

Mean Plot of Class 2:

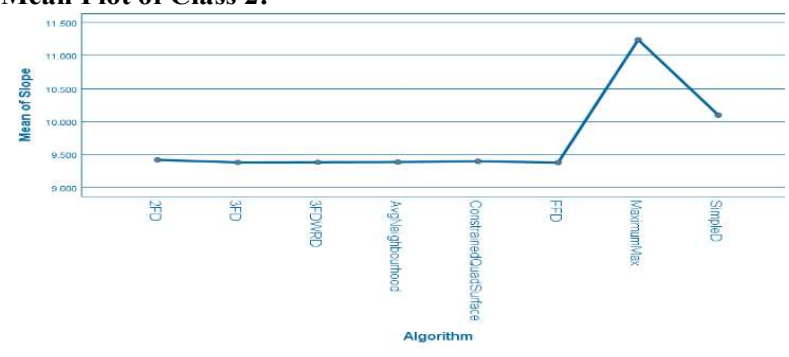

Figure 8. Mean slopes of each algorithm for Class 2

ANOVA Test Class 3: The ANOVA test was significance which indicates that at least two algorithms among the eight algorithms were different for Class 3.

Table 6. ANOVA Test for Class 3

\begin{tabular}{|l|r|r|r|r|c|}
\hline & \multicolumn{1}{|c|}{$\begin{array}{c}\text { Sum of } \\
\text { Squares }\end{array}$} & \multicolumn{1}{c|}{ df } & Mean Square & F & \multicolumn{1}{c|}{ Sig. } \\
\hline Between Groups & 1843.933 & 7 & 263.419 & 72.321 & .000 \\
Within Groups & 638226.747 & 175224 & 3.642 & & \\
Total & 640070.681 & 175231 & & & \\
\hline
\end{tabular}

Post Hoc Test Class 3: Following ANOVA test, post-hoc test was done to find out the difference among the different algorithms. The following table from Tukey's Post-Hoc test show that the slopes calculated using Maximum Max was significantly different from all other algorithms with mean values, significantly lower than the rest of the algorithms while all the other algorithms produced similar results and weren't significantly different from one another.
Table 7. Tukey Test Result for Class 3

\begin{tabular}{|l|c|c|c|}
\hline \multirow{2}{*}{ Algorithm } & & \multicolumn{2}{|c|}{$\begin{array}{c}\text { Subset for alpha } \\
=0.05\end{array}$} \\
\cline { 3 - 4 } & $\mathrm{N}$ & 1 & 2 \\
\hline MaximumMax & 21904 & 12.306 & \\
FimpleD & 21904 & & 12.609 \\
3FD & 21904 & & 12.617 \\
3FDWRD & 21904 & & 12.617 \\
AvgNeighbourhood & 21904 & & 12.617 \\
2FD & 21904 & & 12.617 \\
ConstrainedQuadSurface & 21904 & & 12.618 \\
Sig. & 21904 & & 12.620 \\
& & 1.000 & .999 \\
\hline
\end{tabular}

\section{Mean Plot Class 3:}

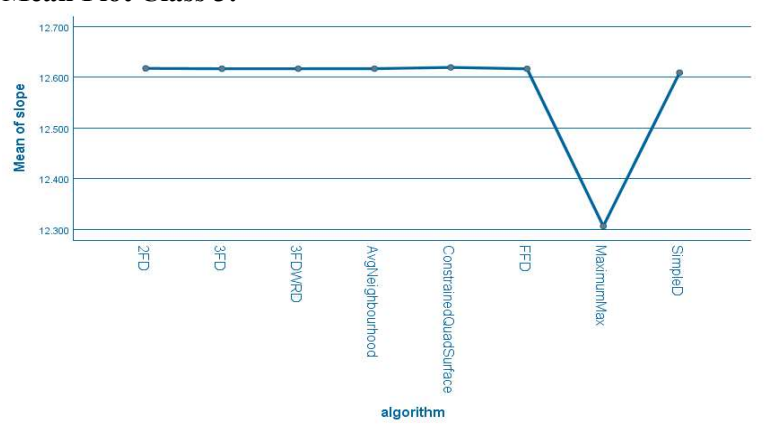

Figure 9. Mean Slope of each algorithm for Class 3

ANOVA Test Class 4: The ANOVA test was insignificant which indicates that no two algorithms were significantly different for Class 4.

Table 8. ANOVA Test Result for Class 4

\begin{tabular}{|l|r|r|r|r|r|}
\hline & \multicolumn{1}{|c|}{$\begin{array}{c}\text { Sum of } \\
\text { Squares }\end{array}$} & \multicolumn{1}{c|}{ df } & Mean Square & \multicolumn{1}{c|}{ F } & Sig. \\
\hline Between Groups & 4.168 & 7 & .595 & .040 & 1.000 \\
Within Groups & 2612960.183 & 175224 & 14.912 & & \\
Total & 2612964.351 & 175231 & & & \\
\hline
\end{tabular}

Post Hoc: The post-hoc wasn't required for class 4 because the ANOVA was insignificant. The results that the algorithms produced weren't significantly different from one another.

\section{Mean Plot Class 4:}

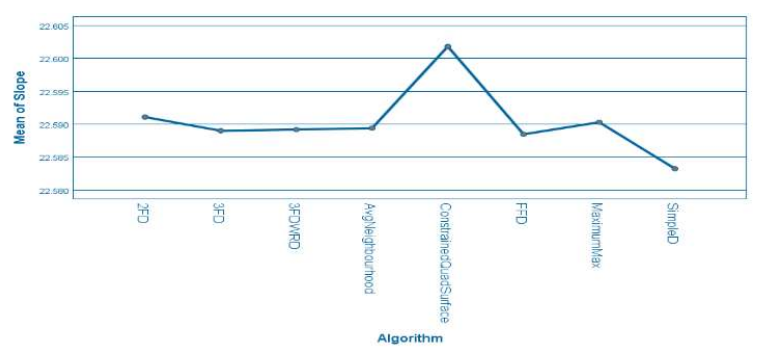

Figure 10. Mean slope of each algorithm for Class 4

ANOVA Test Class 5: The ANOVA test was significance which indicates that at least two algorithms among the eight algorithms were different for Class 5 . 
Table 9. ANOVA Test Result for Class 5

\begin{tabular}{|l|r|r|r|r|r|}
\hline & \multicolumn{1}{|c|}{$\begin{array}{c}\text { Sum of } \\
\text { Squares }\end{array}$} & \multicolumn{1}{c|}{ df } & Mean Square & F & Sig. \\
\hline Between Groups & 1123.786 & 7 & 160.541 & 2.750 & .007 \\
Within Groups & 10230207.092 & 175224 & 58.384 & & \\
Total & 10231330.878 & 175231 & & & \\
\hline
\end{tabular}

Post Hoc Test Class 5: Following ANOVA test, post-hoc test was done to find out the difference among the different algorithms. The following table from Tukey's Post-Hoc test show that the slopes calculated using Maximum Max was significantly different from all other algorithms with mean values, significantly lower than the rest of the algorithms while the rest of the algorithms had no significant differences.

Table 10. Tukey Test Result for Class 5

\begin{tabular}{|l|c|c|c|}
\hline & & \multicolumn{2}{|c|}{$\begin{array}{r}\text { Subset for alpha } \\
=0.05\end{array}$} \\
\cline { 3 - 4 } Algorithm & $\mathrm{N}$ & 1 & 2 \\
\hline MaximumMax & 21904 & 37.153 & \\
FFD & 21904 & & 37.392 \\
3FD & 21904 & & 37.393 \\
3FDWRD & 21904 & & 37.394 \\
AvgNeighbourhood & 21904 & & 37.394 \\
SimpleD & 21904 & & 37.396 \\
2FD & 21904 & & 37.399 \\
ConstrainedQuadSurface & 21904 & & 37.399 \\
Sig. & & 1.000 & 1.000 \\
\hline
\end{tabular}

\section{Mean Plot Class 5:}

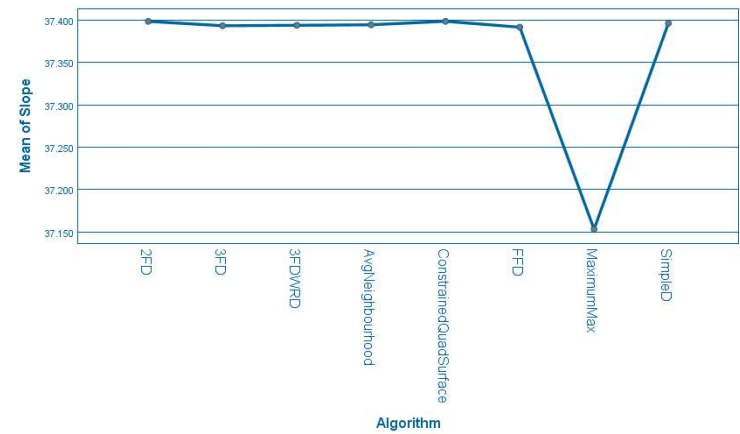

Figure 11. Mean slope of each algorithm for Class 5

ANOVA Test Class 6: The ANOVA test was significance which indicates that at least two algorithms among the eight algorithms were different for Class 6 algorithms while the rest of the algorithms had no significant differences.

Table 11. ANOVA Test Result for Class 6

\begin{tabular}{|l|r|r|r|c|c|}
\hline & \multicolumn{1}{|c|}{$\begin{array}{c}\text { Sum of } \\
\text { Squares }\end{array}$} & \multicolumn{1}{c|}{ df } & Mean Square & F & \multicolumn{1}{c|}{ Sig. } \\
\hline Between Groups & 102205.862 & 7 & 14600.837 & 170.910 & .000 \\
Within Groups & 14969418.583 & 175224 & 85.430 & & \\
Total & 15071624.444 & 175231 & & & \\
\hline
\end{tabular}

Post Hoc Test Class 6: Following ANOVA test, post-hoc test was done to find out the difference among the different algorithms. The following table from Tukey's Post-Hoc test show that the slopes calculated using Maximum Max was significantly different from all other algorithms with mean values, significantly lower than the rest of the algorithms

Table 12. Tukey Test Result from Class 6

\begin{tabular}{|l|c|c|c|}
\hline \multirow{2}{*}{ Algorithm } & & \multicolumn{2}{|c|}{$\begin{array}{c}\text { Subset for alpha } \\
=0.05\end{array}$} \\
\cline { 3 - 4 } & $\mathrm{N}$ & 1 & 2 \\
\hline MaximumMax & 21904 & 83.638 & \\
ConstrainedQuadSurface & 21904 & & 85.919 \\
SimpleD & 21904 & & 85.929 \\
FFD & 21904 & & 85.954 \\
3FD & 21904 & & 85.955 \\
3FDWRD & 21904 & & 85.956 \\
AvgNeighbourhood & 21904 & & 85.956 \\
2FD & 21904 & & 85.958 \\
Sig. & & 1.000 & 1.000 \\
\hline
\end{tabular}

Mean Plot Class 6:

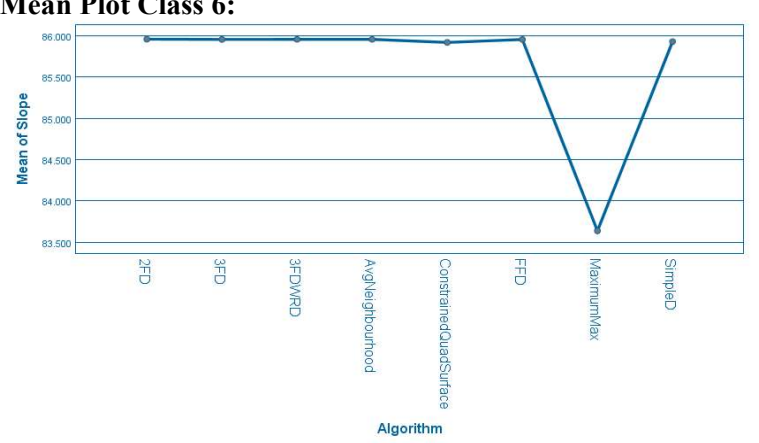

Figure 12. Mean Slope of each algorithm for Class 6

\subsection{Suitability Assessment}

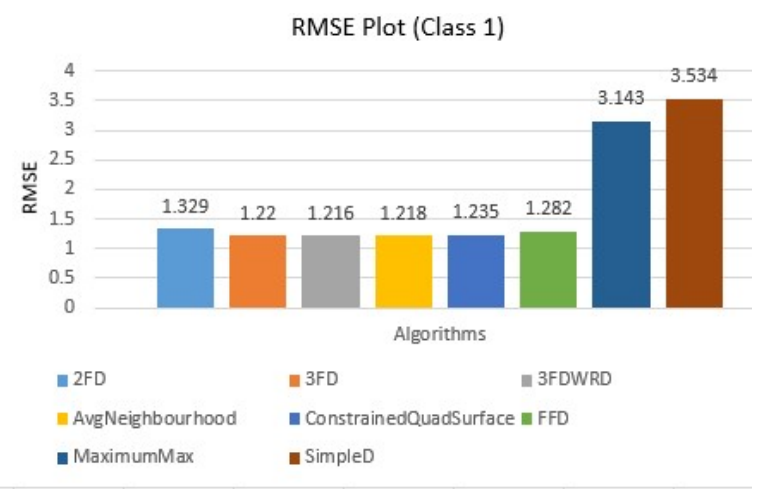

Figure 13. RMSE plot for slope class 1 


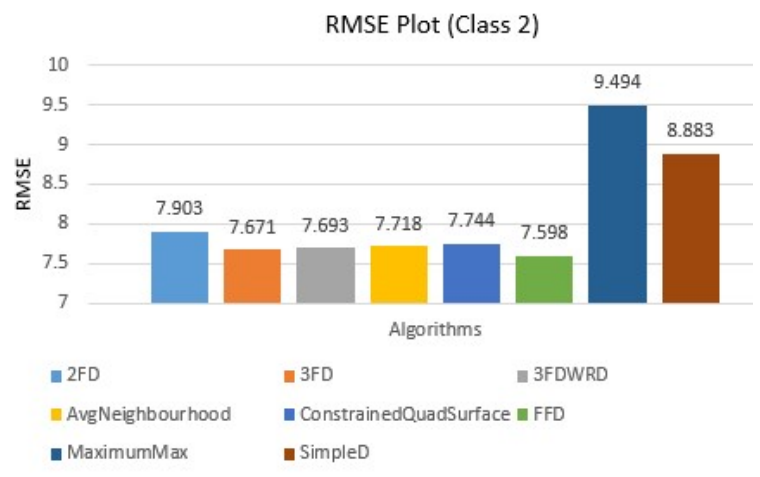

Figure 14. RMSE Plot for slope class 2

RMSE Plot (Class 3)

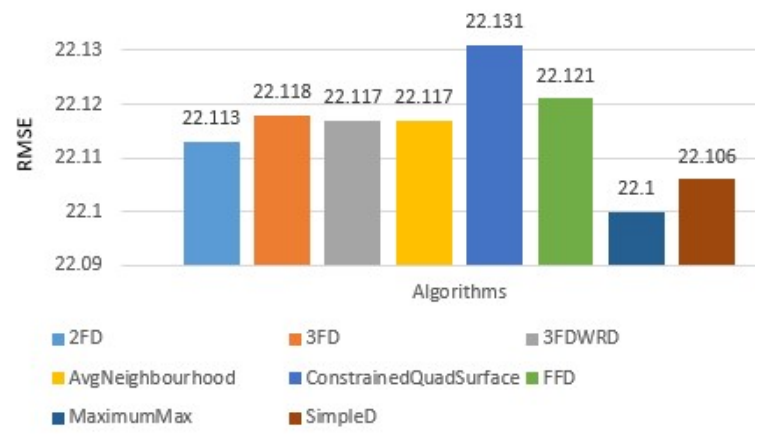

Figure 15. RMSE Plot for slope class 3

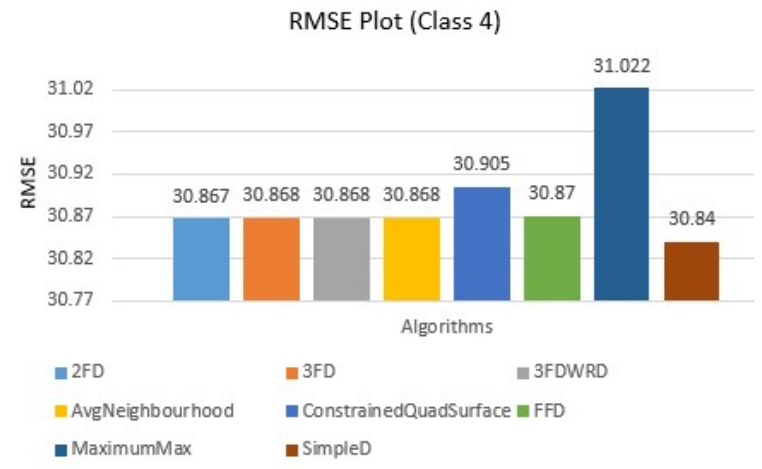

Figure 16. RMSE Plot for slope class 4

RMSE Plot (Class 5)

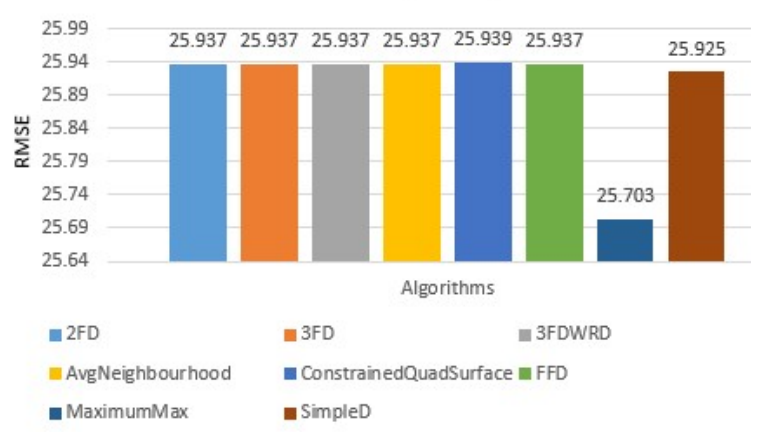

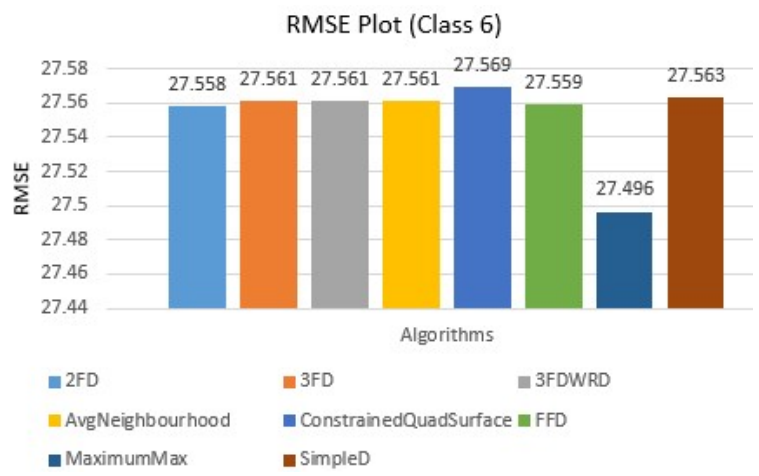

Figure 18. RMSE Plot for slope class 6

The suitability assessment for the algorithms depends on their RMSE value. The RMSE was calculated using the mean slope for each algorithm and ground calculated slope. Algorithms with the lowest RMSE value was the most suitable algorithm for that slope class and the algorithm with largest RMSE was the least suitable algorithm for that slope class. The RMSE plot above represents the RMSE for each algorithm while applying in each slope class. Thus, suitability was derived from the plot and algorithms and has been ranked according as the suitability.

\section{CONCLUSION}

The analysis of slope algorithms was done in two different ways; comparison among the results of the slope algorithms and RMSE calculation of each algorithm's result with the ground measured slopes. The assessment of slope calculation algorithm was done for six different slope classes. Following are the conclusions obtained for each of the classes.

\section{Class 1(0 - 4 grade slope)}

From the ANOVA and post-hoc test, it is seen that all algorithms except Maximum Max and Simple D gives similar results to the rest of the algorithms. The values for those two were drastically higher than the other algorithms. From the RMSE calculation result, the 3FDWRD produces the least error and simple D produces the highest error. Thus, the best algorithm for this class is 3FDWRD.

\section{Class 2(4 - 10 grade slope)}

Similar to class 1 result, it is seen that all algorithms except Maximum Max and Simple D gives similar results to the rest of the algorithms from the ANOVA test. The values for those two were drastically higher than the other algorithms. From the RMSE calculation result, the FFD produced the least error and Maximum Max produced the highest error. Thus, the best algorithm for this class is FFD.

\section{Class 3(10 - 15 grade slope)}

From the ANOVA and post-hoc test, it is seen that all algorithms except Maximum Max gives similar result. The values for those Maximum Max were slightly lower than the other algorithms. However, the difference among all other algorithms aren't statistically significant. From the RMSE calculation result, the Maximum Max produced the least error and Constrained Quadratic Surface produced the highest error. Thus, the best algorithm for this class is Maximum

Figure 17. RMSE Plot for slope class 5 
Class 4(15-30 grade slope)

From the ANOVA and post-hoc test, it is seen that all algorithms give similar result and theirs is no significant difference among algorithms. However, values for those Simple D were slightly lower than the other algorithms and the values for Constrained Quadratic Surface were slightly lower than the other algorithms. From the RMSE calculation result, the 2FD produced the least error and Maximum Max produced the highest error even though all of the RMSE were similar to each other. Thus, the best algorithm for this class is 2FD.

\section{Class 5(30 - 60 grade slope)}

From the ANOVA and post-hoc test, there is significant differences among algorithms though they are small. Values for the Maximum Max algorithm were slightly lower than the other algorithms. From the RMSE calculation result, all the algorithms have similar resulting slopes. However, Maximum Max produced the least error and Constrained Quadratic Surface gives the highest error. Thus, the best algorithm for this class is Maximum Max.

\section{Class 6(60 above grade slope)}

From the ANOVA and post-hoc test, there is significant differences among algorithms though they are small. However, values for those Maximum were slightly lower than the other algorithms. From the RMSE calculation result, all the algorithms have similar resulting slopes. However, Maximum Max produced the least error and Simple D gives the highest error. Thus, the best algorithm for this class is Maximum Max.

The algorithms 2FD, 3FD, 3FDWRD, Average Neighborhood and Constrained Quadratic Surface and FFD has given similar results across all observation while the algorithms that has been observed to producing varying results are Maximum Max and Simple D. In addition to this, it is observed from the above results that the choice of algorithms is more important when grade slope is less than 10 percent. However, for terrains with above 10 percent slope, the choice of algorithms seems less important with only a difference of approximately 0.5 gradient slopes. However, the results from Simple D and Maximum Max has been observed to be more fluctuated. This may be due to the fact that among all algorithms these two are the only algorithms that calculate center cell to adjacent cell slope. Even among these two maximum maxes is the only algorithm that doesn't calculate the $\mathrm{x}$-slope-component and $\mathrm{y}$ slope-component like all the other algorithms and just takes the max slope out of the slopes calculated from center to all surrounding cells.

The conclusions drawn from this project can be used for any general slope calculation purposes. That may include disaster management, hydrological and environmental analysis, suitability assessment, construction and land trainings and other similar projects.

\section{REFERENCES}

Dr. Ashraf, I., 2012. GIS-evaluation of two slope-calculation methods regarding their suitability in slope analysis using high-precision LiDAR digital elevation models. Hydrological Processes, 1119-1133.

Fleming, M. D., \& Hoffer, R. M., 1979. Machine Processing of Landsat MSS Data and DMA Topographic Data for Forest
Cover Type Mapping. Machine Processing of Remotely Sensed Data Symposium, 377-390.

Horn, B.,1981, February 19. Hill Shading and the reflectance map. Proceedings of the IEEE, 14-47. Retrieved July 21, 2019

Jones, H. K., 1998. A Comparison of Two Approaches to Ranking Algorithms Used to Compute Hill Slopes. GeoInformatica, pp. 315-323.

Sharpnack, D., \& A. and AKin, G., 1969. An algorithm for computing slope and aspect from elevations. Photogrammetry Survey 35, 247-248.

Stevens., 1999. Posthoc Test ANOVA. Applications of Statistical Tests, V, 103-148. Retrieved August 24, 2019

Unwin, D., 1981. Introductory Spatial Analysis. Methuen, London and New York, 12, 819-820. Retrieved July 30, 2019

Wood, J., 1996. The geomorphological characterisation of Digital Elevation Models. Theses, Dept. of Geography Leiciester Theses. 